\title{
TOPOLOGICAL ENTROPY AND PERIODS OF GRAPH MAPS
}

\author{
JAUME LLIBRE AND RADU SAGHIN
}

\begin{abstract}
We give a complete description of the set of periods of continuous self-maps on graphs which have zero topological entropy and all their branching points are fixed. As a corollary we get a criterion for positive topological entropy for continuous self-maps on graphs. We also prove that the results are optimal.
\end{abstract}

\section{INTRODUCTION}

If a function $f$ is a self-map on some space $M$, then one of the simplest dynamical invariants one would like to describe is the set of periodic points. A point $x \in M$ is periodic if there exists $n \in \mathbb{N}$ such that $f^{n}(x)=x$, and the smallest $n$ with this property is called the (least) period of $x$. The set of periods of $f$ is

$\operatorname{Per}(f)=\{n \in \mathbb{N}: n$ is the period of some periodic point $x$ of $f\}$.

This set is well understood in the case of continuous maps on some simple spaces like the interval $([9])$, circle ([1] for example), 'Y' $([2]), n$-odd ([3]), trees with branching points fixed ([4]). However if the space becomes more complicated, or higher dimensional, it is very difficult to describe the set of periods for a general map, so one usually has to add some restrictions.

If $M$ is a metric space and $f$ is continuous, then one can define the topological entropy of $f$, or simply the entropy of $f$, denoted $h(f)$, as an invariant measuring the complexity of the orbits of $f$ (see [7] for example). The presence of positive entropy implies chaotic behavior, so one would like to understand when this happens.

A graph is a union of vertexes - points - and edges, which are homeomorphic to the cloesed interval, and have mutually disjoint interiors. The endpoints of the edges are vertexes (not necessarily different). An endpoint of a graph is a vertex which is the endpoint of only one edge. A branching point is a vertex which is the endpoint of at least three edges (if an edge has both endpoints at that vertex, we count the edge twice). An ending edge is an edge which contains an endpoint of the graph. A path in the graph $G$ is the image of a continuous map from $[0,1]$ to $G$. If the endpoints coincide, and the path is not homotopic to a point, then it is called a cycle. A tree is a graph which does not contain any cycle. One can put a metric on any graph, first on the edges from the homeomorphism with the interval, then on the entire graph, by taking the length of the shortest path between any two points. The simplest examples of graphs are the interval (one edge, two endpoints), the circle (one edge, one vertex), the $n$-odd (one branching point, $n$ edges, $n$ endpoints). A continuous map from a graph to itself is called a graph map.

1991 Mathematics Subject Classification. Primary: 37E25, 37B40.

Key words and phrases. graph maps, periodic points, periods, topological entropy. 
For continuous one-dimensional maps, the knowledge of the set $\operatorname{Per}(f)$ gives direct information on the topological entropy of $f$, and vice versa. In the case of the interval, positive entropy is equivalent to existence of periodic orbits with least period not a power of two (and equivalently infinitely many of them). For trees, if there is a periodic orbit with the period having the greatest odd divisor greater then some bound depending on the graph, then the topological entropy is positive $([5])$.

If the graph contains cycles, then bounds on the greatest odd divisors of periods are irrelevant for entropy, in their place we have to look at the number of different greatest odd divisors. For example, the rotation by $1 / n$ on the circle has periodic points of least period $n$, and zero entropy. For the circle, if the rotation interval (see [1] for example) has nonempty interior, then the entropy is positive. In fact, if there exist two least periods with different greatest odd divisors, then the entropy is positive. For general graphs, if the number of greatest odd divisors of the numbers in $\operatorname{Per}(f)$ is big enough, then again the entropy must be positive. There are bounds depending on the graph given in [6] and [8], but they are very big, and in general not optimal. Also it is known that if the entropy is positive, then there are infinitely many periodic points (actually infinitely many greatest odd divisors).

We will consider the case when the graph $G$ contain at least one branching point (the cases of the interval and the circle are well understood), and the continuous self-map $f$ on the graph $G$ preserves the branching points and has zero entropy. The assumption that all the branching points are fixed is quite restrictive, but it allows us to give sharp bounds for the greatest odd divisors of the periods (and not only their number) for any graph, which are much smaller than the ones in [6] and [8]. First, we prove that the periods must have a specific form.

Theorem 1.1. Let $G$ be a graph with $v$ vertexes, $s$ edges and e endpoints, at least one branching point, and $f$ a continuous map from $G$ to itself such that all the branching points are fixed and the topological entropy of $f$ is zero. Then for any periodic point $p$ of $f$, there exist integers $1 \leq k \leq e+2 s-2 v+2, k$ is odd, and $l \geq 0$, such that the least period of $p$ is equal to $k 2^{l}$.

We also give a complete description of the possible set of periods of such a self-map $f$.

Theorem 1.2. Let $G$ be a graph with $v$ vertexes, $s$ edges and e endpoints, at least one branching point, and $f$ a continuous map from $G$ to itself such that all the branching points are fixed and the topological entropy of $f$ is zero. Then there exist integers $1 \leq k_{1}<k_{2}<\cdots<k_{r} \leq e+2 s-2 v+2$ and $l_{1}, l_{2}, \ldots, l_{r} \in \mathbb{N} \cup\{\infty\}$ such that the periods of $f$ are

$$
\operatorname{Per}(f)=\{1\} \cup\left\{k_{i} 2^{l}: 1 \leq i \leq r, 0 \leq l<l_{i}\right\} .
$$

Finally, we prove that the result of Theorem 1.2 is sharp.

Proposition 1.3. Let $G$ be a connected graph with $v$ vertexes, s edges and e endpoints. Given any sequence of integers $1 \leq k_{1}<k_{2}<\cdots<k_{r} \leq e+2 s-2 v+2$, and any $l_{1}, l_{2}, \ldots, l_{r} \in \mathbb{N} \cup\{\infty\}$, there exist a continuous map $f$ from $G$ to itself such that the branching points of $G$ are fixed, the topological entropy of $f$ is zero, and the set of periods of $f$ is

$$
\operatorname{Per}(f)=\{1\} \cup\left\{k_{i} 2^{l}: 1 \leq i \leq r, 0 \leq l<l_{i}\right\} .
$$


As a simple corollary we get the following result.

Corollary 1.4. Let $f$ be a continuous map on a graph $G$ with e endpoints, s edges, $v$ vertexes and at least one branching point, which keeps the branching points fixed. Then the topological entropy of $f$ is positive if and only if there exist a periodic orbit of period $n$, where the greatest odd divisor of $n$ is strictly greater than $e+2 s-2 v+2$.

We remark that in the above results one may assume that the vertexes of $G$ are only branching points and endpoints, otherwise one can just replace $G$ with another graph with these properties, which is homeomorphic to $G$. Also the results are the same if we make the extra assumption that the endpoints are also fixed. If the graph is not connected, the assumption that the branching points are fixed implies that the map preserves the connected components, and one can study just the restriction of the map to these components, so we can also assume that the graph is connected, even if this condition is necessary only in Theorem 1.3. The results remain true in the case of the interval, and in the case of the circle if we assume that the vertex is fixed.

In Section 2 we give a description on how the points from a periodic orbit for a map with the required properties are situated on the edges of the graph. In Section 3 we give a bound for the greatest odd divisor of such a period, and complete the proofs of Theorems 1.1 and 1.2. In Section 4 we prove Theorem 1.3.

\section{Periodic Points and Edges}

In this section we will describe how the points from a periodic orbit of a graph map with zero entropy and the branching points fixed must be situated on the edges. First we will make some preliminary definitions and remarks.

Let $p$ be a periodic point of period $n$ and let $P=\left\{p, f(p), \ldots, f^{n-1}(p)\right\}$ be the orbit of $p$. Let $B$ be the set of branching points of $G$. We call an interval the image of an embedding $i:[0,1] \rightarrow G$, with the endpoints $i(0)$ and $i(1)$. If $x \in B$ and $a \in P$ then we say that any interval with the endpoints $x$ and $a$ is a branched interval (if we want to specify that the branching point is $x$, we will call it $x$-branched).

We say that the interval $A$ covers the interval $B$, and we write $A \rightarrow B$, if there exist $i>0$ and an interval $C \subset A$ such that $f^{i}(C)=B$ (if we want to specify $i$ we say that $A i$-covers $B)$. This is a transitive relation, if $A(i)$ covers $B$, and $B(j)$ covers $C$, then $A(i+j)$ covers $C$. A sequence on intervals $A_{1}, A_{2}, \ldots, A_{k+1}=A_{1}$ is called a loop if $A_{i} \rightarrow A_{i+1}$ for all $1 \leq i \leq k$. Two loops starting from the same interval, $A_{1} \rightarrow A_{2} \rightarrow \ldots \rightarrow A_{k} \rightarrow A_{1}$ and $B_{1}=A_{1} \rightarrow B_{2} \rightarrow \ldots \rightarrow B_{l} \rightarrow B_{1}$, are called essentially different, if there exist $1<i \leq k, 1<j \leq l$, and $m>0$, such that $A_{i}$ and $B_{j}$ have disjoint interiors, $A_{1} m$-covers $A_{i}$, and $B_{1} m$-covers $B_{j}$.

The following is a well-known result, which is one of the main tools in the study of the entropy of one-dimensional maps (see [1] for example).

Lemma 2.1. If a continuous map from a graph $G$ to itself has two essentially different loops starting from the same interval, then the topological entropy of $f$ is strictly positive.

In order to simplify the notations, from now on we assume that the only vertexes of $G$ are the branching points and the endpoints. If $S$ is a finite set, then $|S|$ denotes its cardinal. The main result of this section is the following. 
Proposition 2.2. Let $f$ be a continuous self-map of the graph $G$, which has zero entropy and the branching points fixed. Let $P$ be a periodic orbit of $f$ with period $n$. Then there exist integers $l>0$ and $k \geq 0$ such that $n=l 2^{k}$, and for each edge $E \subset G$ we have exactly one of the following three possibilities:

- $E \cap P=\emptyset$ (there are no points from the periodic orbit on $E$ );

- $|E \cap P|=2^{k}$, and for any $i>0$, there exists an edge $E_{i}$, such that $f^{i}(E \cap$ $P) \subset E_{i}$ (there are $2^{k}$ points from the periodic orbit on $E$, and they always stay on the same edge when we iterate them);

- $E \cap P=P_{E}^{1} \cup P_{E}^{2},\left|P_{E}^{1}\right|=\left|P_{E}^{2}\right|=2^{k}, P_{E}^{1}$ and $P_{E}^{2}$ are disjoint, included in two disjoint intervals, and for every $i>0$, there exist edges $E_{i}^{1}$ and $E_{i}^{2}$, such that $f^{i}\left(P_{E}^{1}\right) \subset E_{i}^{1}$ and $f^{i}\left(P_{E}^{2}\right) \subset E_{i}^{2}$ (there are two disjoint subintervals in $E$, each one containing $2^{k}$ points from the periodic orbit, and the points from each one of these two subintervals always stay on the same edges when we iterate them).

Furthermore, an ending edge cannot be of the third type.

Before proving Proposition 2.2 we need several lemmas.

Lemma 2.3. Let $f$ be a continuous self-map on the graph $G$, with the branching points fixed, $P$ a periodic orbit, $E$ an edge between a branching point and an endpoint, and assume that $a, b \in P \cap E$. If there exist $i>0$ such that $f^{i}(a)$ and $f^{i}(b)$ belong to different edges, then $h(f)>0$.

Proof. Let $x$ be the branching point bounding $E$, and assume that the order inside $E$ is $x-a-b$. Let $A$ be the interval with endpoints $x$ and $b$. Because $f^{i}(a)$ and $f^{i}(b)$ are on different edges, $f^{i}(A)$ must contain two branched intervals with disjoint interiors, $A_{1}=\left[z_{1}, f^{i}(a)\right]$ and $A_{2}=\left[z_{2}, f^{i}(b)\right]$. There exist $j, k \geq 0$ such that $f^{i+j}(a)=b$ and $f^{i+k}(b)=b$. This implies that both $A_{1}$ and $A_{2}$ will cover $A$, so there are two essentially different loops starting from $A$, and hence, by Lemma $2.1, h(f)>0$.

Lemma 2.4. Let $f$ be a continuous self-map on the graph $G$, with the branching points fixed, $P$ a periodic orbit, $E$ an edge between two branching points, and assume that $a, b, c \in P \cap E$ are in this order inside an edge $E$. If there exist $i, j>0$ such that $f^{i}(a)$ and $f^{i}(b)$ are on different edges, and $f^{j}(b)$ and $f^{j}(c)$ are also on different edges, then $h(f)>0$.

Proof. Let $x$ and $y$ be the branching points bounding $E$, and assume we have the order $x-a-b-c-y$. Let $A$ be the interval between $a$ and $b$ inside $E$, and $B$ the interval between $b$ and $c$. Then $f^{i}(A)$ will be a path from $f^{i}(a)$ to $f^{i}(b)$. Because $f^{i}(a)$ and $f^{i}(b)$ are in different edges, this path must contain two branched intervals with disjoint interiors $A_{1}=\left[f^{i}(a), z_{1}\right]$ and $A_{2}=\left[f^{i}(b), z_{2}\right]$, so $A \rightarrow A_{1}$ and $A \rightarrow A_{2}$ $\left(z_{1}\right.$ and $z_{2}$ are branching points, not necessarily different). There exists $j \geq 0$ such that $f^{i+j}(a)=b$, so $f^{j}\left(A_{2}\right)$ is a path from $z_{1}$ to $b$, so it has to contain either $A$ or $B$. The same argument shows that $A_{2}$ also has to cover either $A$ or $B$. In the same way we can prove that $B$ covers two intervals with disjoint interiors $B_{1}$ and $B_{2}$, and each one of these two intervals has to cover either $A$ or $B$. It is an easy exercise to show that this would imply the existence of two essentially different loops starting from $A$ or $B$, and hence strictly positive entropy. 
Lemma 2.5. Let $f$ be a continuous self-map on the graph $G, E$ an edge in $G$ with the endpoints fixed, and $P$ a periodic orbit for $f$ inside $E$, with period $m$. If $h(f)=0$ then $m$ must be a power of two.

Proof. Let $a$ and $b$ be the endpoints of $E$. Let $\mathbb{S}^{1}=\mathbb{R} / \mathbb{Z}$, and $i:[0,1) \rightarrow E$ be a continuous function homeomorphic to $E \backslash\{b\}$. Using the standard identification of $\mathbb{S}^{1}$ with $[0,1)$, we will also denote by $i$ the map from $\mathbb{S}^{1}$ to $E$, which may have an one-sided discontinuity at 0 . Let $p: G \rightarrow \mathbb{S}^{1}$ be the continuous map given by $p(x)=i^{-1}(x)$ if $x \in E \backslash\{b\}$, and $p(x)=0$ otherwise. Let $g: \mathbb{S}^{1} \rightarrow \mathbb{S}^{1}$ be defined by $g=p \circ f \circ i$. Since $a$ and $b$ are fixed by $f$, it is easy to see that $g$ is a continuous map of the circle, and $f$ is semi-conjugated to $g$ by $p$. Since $h(f)=0$, we have $h(g)=0$ too. The point 0 is fixed for $g$, and $p(P)$ is a periodic orbit of $g$ of period $m$. For continuous maps of the circle is well known that if the topological entropy is zero, then every period must be of the type $l 2^{k}$ for some unique $l$ (see [1] for example). In our case $l$ must be one, so $m$ must be a power of two.

Now we will give the proof of Proposition 2.2.

Proof of Proposition 2.2. Let $E$ be an edge, which contains $m^{\prime}>0$ points of the periodic orbit $P$. Let $P \cap E=P_{E}$. Then by Lemmas 2.3 and 2.4, we have two possibilities.

Case 1. The points in $P_{E}$ never separate under the iterates of $f$, meaning that $f^{i}\left(P_{E}\right)$ is contained in the same edge for any $i \geq 0$. Assume that there exist $0 \leq i_{1}<i_{2}<i_{3}<n$ and an edge $E^{\prime}$ such that $f^{i_{s}}\left(P_{E}\right)=P_{E^{\prime}}^{s} \subset E^{\prime}$, for $1 \leq s \leq 3$. We have that $f^{n-i_{s}}\left(P_{E^{\prime}}^{s}\right)=P_{E}$ and $f^{n-i_{s}}\left(P_{E^{\prime}}^{r}\right)$ is inside an edge different from $E$, for $1 \leq s, r \leq 3, s \neq r$. If we choose $x_{s} \in f^{i_{s}}\left(P_{E}\right), 1 \leq s \leq 3$, we can assume that they are in this order in $E^{\prime}$, and it is easy to see that $f^{n-i_{1}}\left(x_{1}\right) \in E$, while $f^{n-i_{1}}\left(x_{2}\right) \notin E$, and $f^{n-i_{2}}\left(x_{2}\right) \in E$, while $f^{n-i_{2}}\left(x_{3}\right) \notin E$. This contradicts Lemma 2.4 .

This means that for any edge $E^{\prime}$, there are at most two indexes $0<i, j<n$ such that $f^{i}\left(P_{E}\right), f^{j}\left(P_{E}\right) \subset E^{\prime}$ and $f^{i}\left(P_{E}\right) \cap f^{j}\left(P_{E}\right)=\emptyset$. As a consequence on any edge there can be zero, $m=m^{\prime}$ or $2 m$ points from $P$. The other claims follow easily.

Case 2. The points in $P_{E}$ separate exactly once under the iterates of $f$, meaning that there exist $0<i<n$ such that $P_{E}=P_{E}^{1} \cup P_{E}^{2}$ and $f^{i}\left(P_{E}^{1}\right)$ and $f^{i}\left(P_{E}^{2}\right)$ are on different edges. Because of Lemma 2.4, $P_{E}^{1}$ and $P_{E}^{2}$ must be inside two disjoint subintervals of $E$, and the points inside them will never separate again under the iterates of $f$. There exist $0<i_{1}, i_{2}<n$ such that $f^{i_{1}}\left(P_{E}^{1}\right) \subset P_{E}^{2}$ and $f^{i_{2}}\left(P_{E}^{2}\right) \subset P_{E}^{1}$, so $\left|P_{E}^{1}\right|=\left|P_{E}^{2}\right|=m=m^{\prime} / 2$. Like in the previous case we obtain that any edge can contain zero, $m$ or $2 m$ points from $P$, and the other claims follow.

The fact that an ending edge can contain at most $m$ points follows from Lemma 2.3. The fact that $m=2^{k}$ for some $k \geq 0$ follows from Lemma 2.5 applied to an edge containing points of $P$ and $f^{l}$, where $l=n / m$.

\section{Periodic Points And Sub-trees}

In this section we will give a bound for the $l$ which appears in the formula $n=l 2^{k}$ of the statement of Proposition 2.2. We start with a simple lemma concerning intervals covering each other for maps with zero entropy.

Lemma 3.1. Let $\mathcal{F}$ be a finite family of intervals with mutually disjoint interiors inside the graph $G$, and $f$ a continuous self-map on $G$ with zero entropy. Then 
there exists an interval $A$ in $\mathcal{F}$ such that any iterate of $A$ will cover at most one of the intervals from $\mathcal{F}$.

Proof. Assume the claim is not true, for any interval in $\mathcal{F}$, some iterate of it covers two intervals with disjoint interiors in $\mathcal{F}$. Let $A_{1} \in \mathcal{F}$. Then there exist different $B_{1}, C_{1} \in \mathcal{F}$ such that $A_{1} \rightarrow B_{1}$ and $A_{1} \rightarrow C_{1}$. If both $B_{1}$ and $C_{1}$ cover $A_{1}$, then there exist two essentially different loops starting from $A_{1}$, and hence positive entropy, which is a contradiction. This implies that at least one of the intervals $B_{1}$ and $C_{1}$ does not cover $A_{1}$, we denote this interval by $A_{2}$. Clearly $A_{2}$ is different from $A_{1}$. Repeating the argument, we can find a new interval $A_{3} \in \mathcal{F}$ such that $A_{2} \rightarrow A_{3}$ and $A_{3}$ does not cover $A_{2}$. in this way we can construct an infinite sequence of different intervals in $\mathcal{F}, A_{1} \rightarrow A_{2} \rightarrow A_{3} \rightarrow \ldots$, which contradicts the fact that $\mathcal{F}$ is finite.

We say that the graph $G^{\prime}$ is a subgraph of $G$ if $G^{\prime} \subset G$ (set inclusion). We also have the following result concerning the endpoints of $G^{\prime}$.

Lemma 3.2. Let $G$ be a connected graph with e endpoints, $v$ vertexes and $s$ edges, and $G^{\prime}$ a connected subgraph of $G$. Then the number of endpoints of $G^{\prime}$ is smaller or equal than $e+2 s-2 v+2$.

Proof. By eventually removing some edges between branching points in $G^{\prime}$, without changing the number of endpoints, we can assume that $G^{\prime}$ is a connected tree. Given a loop $L \subset G$ (a closed path in $G$ which is not homotopic to a point), there exists a point $x_{1} \in L \backslash G^{\prime}$, because otherwise $G^{\prime}$ would contain a loop and it would not be a tree. Because $G^{\prime}$ is closed, there exists a small open interval $I_{1}$ around $x_{1}$ contained in $L \backslash G^{\prime}$, and eventually taking a subinterval, we may assume $I_{1}$ is strictly inside an edge of $G$. Let $G_{1}=G \backslash I_{1}$, be the subgraph of $G$, containing $G^{\prime}$, and having $e+2$ endpoints, $s+1$ edges and $v+2$ vertexes. We can construct inductively in this way subgraphs $G_{i}$ of $G$, containing $G^{\prime}$, with $e+2 i$ endpoints, $s+i$ edges and $v+2 i$ vertexes, as long as there are still loops available, until we obtain a tree $T$. This happens when the number of edges minus the number of vertexes is -1 , or $s+i-v-2 i=-1$, or $i=s-v+1$. Consequently $T$ will contain $G^{\prime}$ and will have $e+2 s-2 v+2$ endpoints, which means that the number of endpoints of $G^{\prime}$ must be at most $e+2 s-2 v+2$.

Proposition 3.3. Let $f, G, P, n, l, k$ be as in Proposition 2.2. Then $l \leq e+2 s-$ $2 v+2$

Proof. Let $\mathcal{F}$ be a family of intervals with mutually disjoint interiors in $G$ defined in the following way. If $\mathrm{E}$ in an ending edge which contains points from $P, x$ is the branching point bounding $E$, and $a$ is the point in $E \cap P$ which is the closest to $x$, then we take the interval $[a, x]$. If $E$ is an edge between the branching points $x$ and $y$, containing points from $P$, and $a$ and $b$ are the points from $E \cap P$ which are closest to $x$ and $y$ respectively, then we take the intervals $[a, x]$ and $[b, y]$. The collection of all the intervals obtained in this way will be the family

$$
\mathcal{F}=\left\{\left[a_{i}, x_{i}\right], a_{i} \in P, x_{i} \text { is a branching point, } 0 \leq i \leq l^{\prime}-1\right\} .
$$

It is possible to have $a_{i}=a_{j}$ or $x_{i}=x_{j}$ for some different indexes $i$ and $j$. We can express $l$ and $l^{\prime}$ in the following way. Let $\epsilon$ be the number of ending edges containing $m=2^{k}$ points of $P, \beta_{1}$ the number of edges between branching points 
containing $m$ points of $P$, and $\beta_{2}$ the number of edges between branching points containing $2 m$ points of $P$. Then $l=\epsilon+\beta_{1}+2 \beta_{2}$ and $l^{\prime}=\epsilon+2 \beta_{1}+2 \beta_{2}$. The family $\mathcal{F}$ satisfies the hypothesis of Lemma 3.1 , so there exists an interval $\left[a_{0}, x_{0}\right] \in \mathcal{F}$ such that $f^{n_{i}}\left(\left[a_{0}, x_{0}\right]\right)$, which is a path from $x_{0}$ to $f^{n_{i}}\left(a_{0}\right)=a_{i}$, covers at most (actually exactly) one interval from $\mathcal{F}$, for every $i \geq 0$. This implies that there exists a path $\gamma_{i}$ in $G$ which is homotopic to $f^{n_{i}}\left(\left[a_{0}, x_{0}\right]\right)$, with endpoints $x_{0}$ and $a_{i}$, which contains either the interval $\left[x_{i}, a_{i}\right]$ or $\left[x_{j}, a_{i}\right]$ (where $x_{j}$ is the other branching point bounding the edge containing $a_{i}$; does not exist for ending edges), and is disjoint from the interior of any other interval from $\mathcal{F}$.

Let $G^{\prime}$ be the union of $\left[a_{0}, x_{0}\right]$ and the paths $\gamma_{i}$, so it is a connected subgraph of $G$. We remark that $x_{0}$ cannot be an endpoint of $G^{\prime}$, otherwise some $\gamma_{i}$ will contain both $\left[a_{0}, x_{0}\right]$ and a branched interval with an endpoint $a_{i}$, which contradicts the choice of $\left[a_{0}, x_{0}\right]$ (we assume that $m \geq 2$, otherwise the conclusion of the proposition is obviously true). This means that candidates for the endpoints of $G^{\prime}$ are $a_{i}$, for $0 \leq i \leq l^{\prime}-1$.

Unfortunately in general the number of endpoints of $G^{\prime}$ may be smaller than $l^{\prime}$ and even $l$, so we will have to construct another subgraph $G^{\prime \prime}$, which will have at least $l$ endpoints. This will be done by replacing each path $\gamma_{i}$ by another one, $\gamma_{i}^{\prime}$, in the following way.

If $\left[a_{i}, x_{i}\right]$ is an interval in an ending edge, and $i \neq 0$, then $a_{i}$ must be an ending point of $G^{\prime}$. In this case we let $\gamma_{i}^{\prime}=\gamma_{i}$.

Now suppose that $\left[x_{i}, x_{j}\right]$ is an edge between branching points, containing the two intervals $\left[a_{i}, x_{i}\right]$ and $\left[a_{j}, x_{j}\right]$ from $\mathcal{F}$. If $a_{i}=a_{j}$, then $m=1$ and the edge $\left[x_{i}, x_{j}\right]$ contains only one point from $P\left(a_{i}\right)$. In this case $\gamma_{i}$ coincides with $\gamma_{j}$ and $a_{i}$ is an endpoint of $G^{\prime}$. In this case again we let $\gamma_{i}^{\prime}=\gamma_{i}$. If $a_{i} \neq a_{j}$ then we have three possibilities. If $\gamma_{i} \cap \gamma_{j} \cap\left(x_{i}, x_{j}\right)=\emptyset$, then both $a_{i}$ and $a_{j}$ are endpoints of $G^{\prime}$. In this case we let $\gamma_{i}^{\prime}=\gamma_{i}$ and $\gamma_{j}^{\prime}=\gamma_{j}$. If $\gamma_{i} \cap \gamma_{j} \cap\left(x_{i}, x_{j}\right)=\left[a_{i}, a_{j}\right]$, then we replace $\gamma_{i}$ by $\gamma_{i}^{\prime}=\gamma_{i} \backslash\left[a_{i}, a_{j}\right)$, and $\gamma_{j}$ by $\gamma_{j}^{\prime}=\gamma_{j} \backslash\left(a_{i}, a_{j}\right]$ (in this way we create two endpoints for the new subgraph). If $\gamma_{i} \cap \gamma_{j} \cap\left(x_{i}, x_{j}\right)=\left(x_{i}, a_{i}\right]$ (or $\left.\left(x_{j}, a_{j}\right]\right)$, then $G^{\prime}$ has one endpoint in $\left(x_{i}, x_{j}\right.$ ), which is $a_{j}$ (or $a_{i}$ ). In this case we let again $\gamma_{i}^{\prime}=\gamma_{i}$ and $\gamma_{j}^{\prime}=\gamma_{j}$. This situation cannot happen if $\left[x_{i}, x_{j}\right]$ contains $2 m$ points from $P$. This is because $\left[a_{0}, x_{0}\right]$ will cover $\left[a_{i}, a_{j}\right]$, and in turn some iterate of this will contain two branched intervals with disjoint interiors (because they separate), and these branched intervals will eventually cover back $\left[a_{0}, x_{0}\right]$, creating two essentially different loops from $\left[a_{0}, x_{0}\right]$, which would be a contradiction.

Let $G^{\prime \prime}=\cup_{i=1}^{l^{\prime}-1} \gamma_{i}^{\prime} \cup\left[a_{0}, x_{0}\right]$. From the construction it is easy to see that every edge of $G$ containing $m$ points from $P$ will also contain at least one endpoint of $G^{\prime \prime}$, and every edge of $G$ containing $2 m$ points from $P$ will also contain two endpoints of $G^{\prime \prime}$, so the number of endpoints of $G^{\prime \prime}$ is greater or equal than $l$. Then Lemma 3.2 implies that $l \leq e+2 s-2 v+2$.

Now we can give the proofs of Theorems 1.1 and 1.2.

Proof of Theorem 1.1. Apply directly Propositions 2.2 and 3.3.

Proof of Theorem 1.2. Apply Propositions 2.2 and 3.3, and the following lemma, which is a refinement of Lemma 2.5 .

Lemma 3.4. Let $f$ be a continuous self-map on the graph $G, E$ an edge in $G$ with the endpoints fixed, and $P$ a periodic orbit for $f$ inside $E$, with period $2^{k}$. If 
$h(f)=0$ then $E$ contains also periodic points of $f$ with least periods all the divisors of $2^{k}$.

Proof. Let $g$ be the map on the circle constructed in Lemma 2.5, then any period of $g$ is also a period of $f$. Let $\tilde{g}$ be the lift of $g$ to $\mathbb{R}$ which keeps 0 fixed. Suppose that $\tilde{g}^{n}([0,1])$ is not contained in $[-2,2]$, then $\tilde{g}^{n}([0,1])$ must contain either $[-2,0]$ or $[0,2]$, so the interval $[0,1]$ will eventually cover itself twice by $g$, which is a contradiction because the entropy of $g$ must be zero. A similar argument shows that $2^{k}$ must be a period for $\tilde{g}$ also.

So $\cup_{n=0}^{\infty} \tilde{g}^{n}([0,1])=I \subset[-2,2]$ must be a bounded interval, invariant under $\tilde{g}$. Because $2^{k}$ is a period for $\tilde{g}$, Sharkowski order for periods of maps on $\mathbb{R}$ implies that every divisor of $2^{k}$ must also be a period for $\tilde{g}$, and consequently also for $g$ and $f$ (we remark here that a similar argument can be used to reduce the last statement of Lemma 2.5, on periodic points of maps on circle with zero entropy, to the case of interval maps).

\section{MAPS ON GRAPHS WITH PRESCRIBED PERIOdS}

In this section we will prove Proposition 1.3. In the case of the interval and the circle it is well-known, so we will assume again that there is at least one branching point.

Proof of Theorem 1.3. Step 1. It is enough to construct the map $f$ on a connected tree $T$ with $e^{\prime}=e+2 s-2 v+2$ endpoints, obtained from $G$ by removing $s-v+1$ small open intervals inside some edges, with the additional assumption that the endpoints are fixed. From the map on $T$ we can recover the map on $G$ by making it the identity on the removed intervals. The property of having zero entropy is preserved by this transformation, and so are the periodic points, except some extra fixed points for $G$.

Step 2. It is enough to construct the map $f$ on the tree $T$ with periodic points of periods $k_{i}, 1 \leq i \leq r$. In order to construct from this a map with periodic orbits of periods $2^{l} k_{i}$, for $1 \leq l<l_{i}$, one has to 'blow up' each periodic orbit of period $k_{i}$ into a periodic interval, and choose the return map to the interval $\left(f^{k_{i}}\right)$ to have exactly the periods $2^{l}, 1 \leq l<l_{i}$, and with zero entropy (see [1] for example). Again the property of having zero entropy is preserved by this transformation.

Step 3. The construction of $f$ on the tree $T$ with zero entropy and a periodic point of period $k<e^{\prime}$.

Let $\left[x_{i}, y_{i}\right]$ be the ending edges of $T$, with the endpoints $y_{i}$, for $1 \leq i \leq e^{\prime}$. We consider the standard metric $d$ on $T$, such that every edge has length 1 , and the distance between two points is the length of the shortest path between them (the 'taxicab' metric). Choose $k$ points $a_{i} \in\left(x_{i}, y_{i}\right), 1 \leq i \leq k$, such that $d\left(a_{i}, x_{i}\right)=$ $d\left(a_{i}, y_{i}\right)=1 / 2$. On each edge between branching points, and on the ending edges $\left[x_{i}, y_{i}\right]$ for $i>k$, we let $f$ be the identity. On each edge $\left[x_{i}, y_{i}\right], 1 \leq i \leq k$, we define $f$ such that $f\left(x_{i}\right)=x_{i}, f\left(y_{i}\right)=y_{i}, f\left(a_{i}\right)=a_{i+1}$ (with the convention that $k+1=1), f$ maps $\left[a_{i}, y_{i}\right]$ to the shortest path between $y_{i}$ and $a_{i+1}$, expanding the metric by a constant factor $c_{i}>2\left(c_{i}\right.$ is greater than 2 because the length of $\left[a_{i}, y_{i}\right]$ is $1 / 2$, while the length of the path between $y_{i}$ and $a_{i+1}$ is greater than 1 ), and $f$ maps $\left[a_{i}, x_{i}\right]$ to the shortest path between $x_{i}$ and $a_{i+1}$, expanding the metric by a constant factor $d_{i} \geq 1\left(d_{i}\right.$ can be 1 if $\left.x_{i}=x_{i+1}\right)$. 
From construction $f$ has many fixed points, and a periodic orbit with period $k$. We will prove that $f$ has no other periods. We have to analyze if there are other periodic points inside $\left(x_{i}, y_{i}\right)$ for $1 \leq i \leq k$, because all the other points are fixed.

Suppose $x \in\left(a_{i}, y_{i}\right)$. Because $\left.\bar{f}\right|_{\left[a_{i}, y_{i}\right]}$ is expanding by a factor $d>2$, there must be an iterate of $x$ which is outside $\left(a_{i}, y_{i}\right)$, and from the construction of $f$, all the higher iterates of $x$ will never return to $\left(a_{i}, y_{i}\right)$. Consequently $x$ cannot be a periodic point for $f$.

Suppose $x \in\left(a_{i}, x_{i}\right)$. Without loss of generality we can assume that $i=1$. Then $f(x)$ can be either on edges between branching points, in which case further iterates are fixed, or in $\left(a_{2}, x_{2}\right)$. Repeating the argument, we conclude that $f^{k}(x)$ is either on edges between branching points, in which case further iterates are fixed, or it is also in $\left(a_{1}, x_{1}\right)$, in which case we also have $f^{j}(x) \in\left(a_{j}, x_{j}\right)$. Then from the construction we have that $\left.f^{k}\right|_{\left[a_{1}, x\right]}$ expends the metric by a constant factor $d=d_{1} d_{2} \ldots d_{k}$. If $d=1$ then $x$ is a fixed point, so we do not get any new period for $f$. If $d>1$ then there is again an iterate $f^{n}(x)$ which must be on some edge between branching points, so further iterates are fixed, and $x$ cannot be a periodic point for $f$.

We see that the set of periods for $f$ is $\{1, k\}$, so from [8] for example, we get that the entropy of $f$ must be zero, because the set of periods is finite (one can also prove that the non-wandering set consists of fixed points and the periodic orbit of period $k$, so the entropy must be zero).

Step 4. The induction.

We will finish the proof using induction on the number of periods. How to construct a map on the tree $T$ with a periodic orbit of a given period is explained in Step 3. We assume that we have a map $g$ on the tree $T$ with the periods $1, k_{1}, k_{2}, \ldots, k_{j}$, and we will construct another map $f$ on the tree $T$ with the periods $k_{1}, k_{2}, \ldots, k_{j}, k_{j+1}$. Assume we have again a metric on $T$ such that every edge has length one. Let $\left[x_{i}, y, i\right]$ be the ending edges of $T$, and $y_{i}$ the ending points, for $1 \leq i \leq e^{\prime}$. We extend the ending edges by adding to each edge $\left[x_{i}, y_{i}\right]$ a new interval $\left[y_{i}, z_{i}\right]$ of length one, and thus we obtain a new tree $T^{\prime} \supset T$, homeomorphic with $T$, with ending points $z_{i}$.

We construct the map $f$ on $T^{\prime}$ in the following way. On $T$ we let $f=g$, and on $\left[y_{i}, z_{i}\right], k_{j+1} \leq i \leq e^{\prime}$, we let $f$ equal to the identity. Choose $a_{i} \in\left(y_{i}, z_{i}\right)$ such that $d\left(a_{i}, y_{i}\right)=d\left(a_{i}, z_{i}\right)=1 / 2,1 \leq i \leq k_{j+1}$. Let $f\left(z_{i}\right)=z_{i}$ and $f\left(a_{i}\right)=a_{i+1}$, for $1 \leq i \leq k_{j+1}$, with the convention that $k_{j+1}+1=1$. On each interval $\left[a_{i}, y_{i}\right]$ and $\left[a_{i}, z_{i}\right]$, define $f$ such that the image is the shortest path between $a_{i+1}$ and $y_{1}$, respectively $a_{i+1}$ and $z_{i}$, and the metric is expanded by some constant factor greater that one on each interval. Thus we created a periodic orbit of least period $k_{j+1}$ for $f$, and arguments similar as in Step 3 imply that every point in $\left(y_{i}, z_{i}\right) \backslash\left\{a_{i}\right\}$, $1 \leq i \leq k_{j+1}$, after a finite number of iterates will land in $T$, so they cannot be periodic points. This implies that the only periods of $f$ are $1, k_{1}, k_{2}, \ldots, k_{j}, k_{j+1}$, and because of this the topological entropy of $f$ is zero.

To construct the map on $T$, just use a homeomorphism from $T^{\prime}$ to $T$ which maps the intervals $\left[x_{i}, z_{i}\right]$ to $\left[x_{i}, y_{i}\right]$ and keeps all the other edges fixed.

Acknowledgments The first author has been partially supported by MCI/ FEDER grant number MTM 2008-03437 and by CICYT grant number 2009SGR410. The second author has been supported by Marie Curie IEF grant number 234559, and would like to thank CRM Barcelona for their hospitality. 


\section{REFERENCES}

[1] Ll. Alsedà, J. Llibre and M. Misiurewicz, Combinatorial dynamicsm and entropy in dimension one. Second edition. Advanced Series in Nonlinear Dynamics, 5. World Scientific Publishing Co., Inc., River Edge, NJ, 2000.

[2] Alseà, J. Llibre, M. Misiurewicz, Periodic orbits of maps of Y, Trans. Amer. Math. Soc. 313 (1989), no. 2, 475-538.

[3] S. Baldwin, An extension of Šarkovskii's theorem to the n-od, Ergodic Theory Dynam. Systems 11 (1991), no. 2, 249-271.

[4] S. Baldwin and J. Llibre, Periods of maps on trees with all branching points fixed, Ergodic Theory Dynam. Systems 15 (1995), no. 2, 239-246.

[5] A. M. Blokh, Trees with snowflakes and zero entropy maps, Topology 33 (1994), no. 2, 379396.

[6] A. M. Blokh, Spectral decomposition, periods of cycles and a conjecture of M. Misiurewicz for graph maps. Ergodic theory and related topics, III (Gustrow, 1990), 24-31, Lecture Notes in Math., 1514, Springer, Berlin, 1992.

[7] A. Katok and B. Hasselblatt, Introduction to the modern theory of dynamical systems. With a supplementary chapter by Katok and Leonardo Mendoza. Encyclopedia of Mathematics and its Applications, 54. Cambridge University Press, Cambridge, 1995.

[8] J. Llibre and M. Misiurewicz, Horseshoes, entropy and periods for graph maps, Topology 32 (1993), no. 3, 649-664.

[9] O. M. Šarkovskii, Co-existence of cycles of a continuous mapping of the line into itself. (Russian) Ukrain. Mat. Z. 16 (1964), 61-71.

Departament de Matematiques, Universitat Autonoma de Barcelona, Bellaterra, 08193, Catalonia, Spain

E-mail address: jllibre@mat.uab.cat

Centre de Recerca Matematica, Apartat 50, Bellaterra, 08193, Catalonia, Spain

E-mail address: rsaghin@crm.cat 